\section{Heilung eines Falles von Diabetes insipidus durch Bestrahlungen mit ultraviolettem Quarzlampenlicht.}

\section{Von San.-Rat Dr. Hugo Bach in Bad Bertrich.}

Im Juli 1911 wurde folgender Fall von Diabetes insipidus, der auch wegen seiner Aetiologie Interesse bietet, erfolgreich von mir mit ultraviolettem Quarzlampenlicht behandelt.

Anamnese: Herr St., geboren 1874, machte in seinem siebenten bis zwölften Jahre Diptherie, Masern, Wassersucht und Scharlach durch. Während des Scharlachs 1882 litt er an Krämpfen und Schmerzen im rechten Ohr, sowie an Kopfschmerzen. 1886/1887 trat ein AbszeB unterhalb des rechten Warzenfortsatzes auf, der durch Operation beseitigt wurde. Ziemlich gleichzeitig stellten sich Eiterungen aus dem rechten Ohr, sowie starker Kopfschmerz, Schwindel, Erbrechen, Doppeltsehen und Herabsetzung des Empfindungsvermögens in der rechten Gesichtshälfte ein, wodurch im Juli 1888 das Leben des Patienten überaus gefährdet war. In der Klinik des Herrn Prof. Bramann wurde ein eitriger Mittelohrkatarrh im Anschluß an Scharlach und umschriebene Knochenentzündung an der vorderen Wand der Schläfenbeinpyramide in der Nähe des Ganglion Gasseri, sowie sekundäre Vereiterung der Zellen des Warzenfortsatzes mit Eitersenkung an der rechten Halsseite festgestellt und am 30. Juli 1888 von Herrn Prof. Barth operiert. Nach Entleerung des Eiters hörten die bedrohlichen Erscheinungen sofort auf, nur die Empfindungslosigkeit der rechten Gesichtshälfte blieb in derselben Intensität und Ausbreitung bestehen. Die Heilung der Operationswunde verlief zunächst ohne jede Störung, im April $1889 \mathrm{mu}$ ßte aber Herr Prof. Bramann nochmals einen AbszeB an der rechten Halsseite öfnen. Die definitive Ausheilung der Wunde bzw. Fistel erfolgte erst im Juli 1893. Damals war die Gefühlslähmung im Bereiche der rechten Gesichtshälfte noclı vorhanden, auch stellte sich bei stärkerer Anstrengung stets Kopfschmerz ein. Herr Prof. Bramann sprach die Vermutung aus, daß neben den narbigen Schrumpfungen und Gewebsveränderungen möglicherweise noch Reste des ursprünglichen, eitrigen Entzündungsherdes vorhanden wären, die durch Abkapselung im Fortschritte gehindert, aber gelegentlich durch Erschütterung des Schädels oder größere Anstrengung, ja auch ohne jede nachweisbare Ursache sich bemerkbar machen könnten. Patient verlor das Gehör auf dem rechten $\mathrm{Ohr}$ vollkommen, die Lähmung der rechten Gesichtshälfte besserte sich im Laufe der nächsten Jahre durch elektrische Behandlung, das rechte Auge blieb schwach und die Gefühlsempfindung der rechten Seite der Mundschleimhaut und Zunge herabgesetzt, was sich dem Patienten besonders dadurch bemerklich machte, daß er heiße Bissen ohne Schmerzempfindung rechts im Munde auf der Zunge liegen lassen konnte, während sie ihm links Schmerz verursachten.

Die körperliche und geistige Entwicklung des Patienten war jahrelang nach der Operation gehemmt, später normal. Aber seit der Operation, also seit 23 Jahren, leidet Patient Tag und Nacht an einem unstillbaren Durstgefühl, das plötzlich nach der Operation begann. Er trinkt seitde m in 24 Stunden 10-25 Liter Wasser, wovon a uf die Nacht wenigstens vier Liter kommen, ohne da B dadurch das Durstgefühl beseitigt wird. Die Urinmenge ist dem Trinken entsprechend gesteigert, Eiwei $B$ und Zucker wurden nie nachgewiesen. Durch das häufige Trinken und Wasserlassen wird die Nachtruhe stets gestört, wodurch Patient sich morgens matt und abgespannt fühlt. Er leidet außerdem an Kopfschmerzen, sowie an Flimmern auf dem rechten Auge und Gefühllosigkeit der rechten Cornea. Seit Jahren erkrankt er jährlich vier- bis sechsmal an drei- bis achttägigen Fiebern mit Temperaturen bis über $40^{\circ} \mathrm{C}$ und mit Durchfällen, die auf Darmstörungen infolge des vielen Wassertrinkens zurïckgeführt wurden, sowie an heftigen rheumatischen Beschwerden der rechten Körperseite. Er lebt in jeder Beziehung mäßig und erfreut sich, abgesehen von den erwähnten Anfällen, eines leidlich guten Allgemeinbefindens, das ihm gestattet, seinem kaufmännischen Beıufe nachzugehen.

Status am 8. Juli 1911: Patient ist $170 \mathrm{~cm}$ lang und wiegt 85 Kilo. Blasses, volles Gesicht mit vollkommen fehlendem Bartwuchs, reichlichea
Fettpolster, gut entwickelte, schlaffe Muskulatur. Rechts hinter dem Ohr und am Halse tiefe Operationsnarben. Das Mienenspiel der rechten Gesichtshälfte ist langsamer als links. Cornea rechts empfindungslos, Lidschluß rechts verlangsamt und nicht vollkommen, Sehkraft des rechten Auges herabgesetzt. Mundschleimhaut bla $\beta$ und sehr trocken, auf der rechten Seite der Zunge fehlt das Gefühlfürsü $B$, sauer und hei $B$, auf der linken ist es normal. Herztöne rein, Puls $90-100$ Schläge in der Minute, Blutdruck erhöht, Hämoglobingehalt des Blutes $70 \%$, Urin ohne Zucker und Eiweiß. Flüssigkeitsaufnahme in 24 Stunden etwa 10 Liter, Urinmenge dementsprechend gesteigert. Schlaf durch häufiges Trinken und Urinieren gestört. Die Nahrungsmenge pro Tag beträgt nur 13-1400 Kalorien. Patient klagt über ständige Trockenheit im Munde auch nach dem Trinken, sowie über Kopfschmerzen, mangelhaften Appetit, Mattigkeit besonders morgens und über wenig Ausdauer bei der Arbeit. Er sitzt viel in seinem Berufe und lebt fast alkoholfrei. Der Stuhlgang ist zurzeit normal. Ines nicht nachweisbar.

Eine Polyurie, die auf eine Funktionsstörung der Nieren zurück. zuführen ist, wie Erich Meyer sie in seiner Arbeit über Diabetes insipidus im zweiten Bande 1910 der Fortschritte der Medizin kritiseh beleuchtet hat, konnte im vorliegenden Falle von vornherein ausgeschlossen werden, denn die Polyurie des Patienten war unzweifelhaft Folge der Polydipsie. Dás plötzliche Auftreten des unstillbaren Durst gefühls direkt nach der Operation am rechten $\mathrm{Ohr}$ sprach dafür, daß es mit einer Nervenverletzung in der Nähe des Ganglion Gasseri bei der Operation im Zusammenhang stand und Folge der Blässe und Trockenheit der Mundschleimhaut sowie der Gefühlsstörungen der rechten Zungen seite war. Demnach konnte dieser Diabetes insipidus nicht als Krankheit an sich aufgefaßt werden, sondern wurde nur als ein Krankheitssymptom, als eine Folgeerscheinung der Innervationsstörung bezeichnet.

In der Literatur konnte ich keinen Fall von Polydipsie bzw. Diabetes insipidus finden, der in ähnlicher Weise erklärt worden wäre. Erich Me yer, Sally Loewenstein, der in seiner Doktorarbeit (Ueber einen Fall von Diabetes insipidus, Bonn 1904) die Aetiologie einer Anzahl von Diabetes insipidus-Fällen zusammengestellt hat, und andere Autoren konnten zwar mehrfach Gehirn- oder Gehirnnervenerkrankungen als Ursache annehmen, aber auf die Beschaffenheit der Mundhöhlenschleimhaut und Zunge sind sie nicht eingegangen. Eine nähere Erklärung des Zusammenhanges zwischen Diabetes insipidus mit einer Lokalerkrankung fehit in allen Fällen.

In meinem Falle wies das ständige Gefühl des Patienten von Trockenheit im Munde, das trotz vielen Trinkens nicht zu beseitigen war, darauf hin, da $B$ in den Innervationstsörungen der Mundhöhle die Ursache de Diabetes insipidus liegen müßte. Der jahrelange Stillstand der Lokalerkrankung sprach nicht dafür, daß ein Druck auf das Ganglion Gasseri oder benachbarte Nerven durch abgekapselten Eiter in Frage kommen könnte, sondern eher dafür, daß es sich um narbige Veränderungen oder um Druckerscheinungen infolge von Zirkulationsstörungen handeln müßte, die nach Heilung der bei der Operation verletzten Nerven die Polydipsie auslösten.

Die seit Jahren sich wiederholenden Darmstörungen des Patienten mit Fieberanfällen und die rheumatischen Beschwerden wurden als Folgeerscheinungen des vielen Trinkens gedeutet.

Da die bisherige Behandlung, die hauptsächlich in Anwendung von Elektrizität bestand, wohl die Gesichtslähmung gebessert, aber auf die Polydispie keinerlei Einfluß gehabt hatte, wurde zunächst versucht, die Speichelsekretion der trockenen Mundschleimhaut durch Einlegen von Brotrinde oder getrockeneten Pflaumen in den Mund, ähnlich wie Posner es mit Kautabletten vorgeschlagen hat, zu steigern und dadurch die Trockenheit im Munde und das Durstgefühl zu beseitigen. Eine wesentliche Besserung ließ sich sich aber dadurch nicht erzielen.

AuBerdem wurden Bestrahlungen mit ultraviolettem Quarzlampenlicht in der Weise angewandt, wie ich sie in meiner Arbeit in der Deut schen medizinischen Wochenschrift No. 9, 1911 (Einwirkung des ultravioletten Quarzlampenlichtes auf den Blutdruck etc.) näher beschrieben habe. Der Zweck der Bestrahlungen sollte sein, die Zirkulation im Bereiche der Operationsnarben $\mathrm{zu}$ bessern und eventuelle Blutstauungen durch Herabsetzung des Blutdruckes zu beseitigen, sowie den trägen Stoffwechsel anzuregen. Es wurden vom 8.-29. Juli 1911 acht Bestrah lungen des Rückens von 12-20 Minuten Dauer vorgenommen. Schon nach der ersten Bestrahlung verminderte sich das unstillbare Durstgefühl, das den Patienten 23 Jahre lang gequält hatte, und wurde im Laufe der Behandlung vollkommen beseitigt, sodaß Patient trotz de im Juli 1911 herrschenden Hitze bei größeren Spaziergängen und starkem Schwitzen nicht mehr als ein normaler Mensch zu trinken brauchte und dementsprechend weniger urinierte. Der Kopfschmerz, die Darmbeschwerden und rheumatischen Schmerzen schwanden, Patient bekan guten Appetit und Schlaf und fühlte sich frischer und leistungsfähige als seit Jahren. Sein Gewicht, das am 8 . Juli $191185 \mathrm{~kg}$ betrug, fiel am 12. Juli auf $83,8 \mathrm{~kg}$, am 15 . Juli auf 81,9 , am 18 . Juli auf 81,5 , am 21 . Juli auf 80,9 und am 29. Juli auf 80,6, was auf die Entwässerung des Körpers 
zurückgeführt wurde, da Patient mehr Nahrung als bisher zu sich nahm. Der Puls ging von 90-100 auf $70-78$ Schläge in der Minute zurück und war voller und kräftiger als zu Beginn der Kur. Der Blutdruck bewegte sich nach der Behandlung in normalen Grenzen. Die Mundschleimhaut wurde feuchter, das Gefühl für sauer, süß und heiß an der rechten Zungenseite war, wenn auch nur schwach, wieder vorhanden.

Nach der Behandlung mit den ultravioletten Lichtbestrahlungen machte Patient im August 1911 nach Trinken von kaltem Bier eine leichter als früher verlaufende Darmstörung ohne Fieber durch. Es stellte sich vor übergehend, jedoch nicht in dem Maße wie früher, wieder Durst ein, der schon nach drei bis vier Tagen schwand. Seitdem fühlt sich Patient vollkommen gesund und leistungsfähig und kann ohne Ermüdung anstrengende Fußtouren machen. Sein früher auch im Sommer blasses Gesicht ist gebräunt, und seine schlaffe Muskulatur ist straffer geworden. Er genießt ohne Darmbeschwerden die gewöhnliche Hausmannskost, und außer allgemeinen hygienischen Vorschriften besteht die Behandlung nur noch darin, daß er gegen seine Hämoglobinarmut Eisen nimmt. Rheumatische Beschwerden sind nicht wieder aufgetreten, das Gefühl im Munde für sauer, süß und heiß ist rechts fast so wie links, das Gewicht beträgt am 8. September 1911 bei gutem Appetit und Allgemeinbefinden $79 \mathrm{~kg}$.

Wenn Erich Meyer mit anderen Autoren meint, daß der Diabetes insipidus an sich eine wesentliche Schädigung des gesamten Organismus nicht bedingt, so muß ich dem mit Bezug auf den vorliegenden Fall widersprechen. Mein Patient litt unzweifelhaft infolge der Ueberfüllung seines Körpers mit Wasser an Darmstörungen, Hämoglobinarmut und Unterernährung und war dadurch wenig widerstandsfähig gegen $\mathrm{Er}$ krankungen aller Art.

$\mathrm{Ob}$ der überraschende Erfolg der Behandlung mit ultravioletten Lichtbestrahlungen auf Beseitigung von Blutstauungen oder Entzündungsresten oder auf eine bessere Ernährung der verletzten Nerven zurückzuführen ist, entzieht sich der Beurteilung. Es muß aber mit Sicherheit angenommen werden, da $\beta$ nur durch diese Bestrahlungen, und zwar durch ihre blutdruckherabsetzende und stoffwechselsteigernde Wirkung, die Innervationsstörungen und damit die Polydipsie beseitigt wurden. 